\title{
GOULD'S BELT TO STARBURST GALAXIES: THE IMF OF EXTREME STAR FORMATION
}

\author{
M.R. Meyer, J. Greissl, M. Kenworthy, and D. McCarthy \\ Steward Observatory, The University of Arizona, Tucson, AZ 85721-0065 USA \\ mmeyer@as.arizona.edu
}

\begin{abstract}
Recent results indicate the stellar initial mass function is not a strong function of star-forming environment or "initial conditions" (e.g. Meyer et al. 2000). Some studies suggest that a universal IMF may extend to sub-stellar masses (see however Briceno et al. 2002). Yet most of this work is confined to star-forming environments within $1 \mathrm{kpc}$ of the Sun. In order to probe the universality of the IMF over a wider range of parameter space (metalicity, ambient pressure, magnetic field strength) new techniques are required. We begin by summarizing our approach to deriving the sub-stellar IMF down to the opacity-limit for fragmentation using NGC 1333 as an example. Next, we describe results from simulations using the observed point-spread function of the new 6.5m MMT adaptive optics system and examine the confusion-limited sensitivity to low mass stars in rich star-forming clusters out to $0.5 \mathrm{Mpc}$. We also present preliminary results from observations with this system of the W51 star-forming complex. Finally, we outline a new technique to estimate the ratio of high to low mass stars in unresolved stellar populations, such as the massive star clusters observed in interacting galaxies (e.g. Mengel et al. 2002). While evidence for variations in the IMF remains inconclusive, new studies are required to rule them out and determine whether or not the IMF is universal over the range of parameter space relevant to star-forming galaxies over cosmic time.
\end{abstract}

\section{Introduction}

Any predictive theory of star formation must explain the observed shape of the field star IMF (e.g. Chabrier, 2003) and any deviations from it (e.g. Figer et al. 1999) as a function of physical properties. There are a few critical scales in star formation that one might expect should depend on local conditions such as super-critical mass to overwhelm magnetic support (e.g. Shu et al. this volume), or the Jean's mass (e.g. Larson, this volume). Curiously, the stellar IMF observed toward a number of star-forming regions within $1 \mathrm{kpc}$ of the Sun diplay 
no evidence for a strong dependence on "initial conditions" and are consistent with having been drawn from the same IMF that characterizes the field (Meyer et al. 2000). For this reason, we are forced to expand the range of parameter space in which we search for variations in the IMF. There are two obvious avenues for further exploration: 1) probing the sub-stellar IMF in nearby regions down to and below the expected minimum mass for opacity-limited fragmentation (e.g. $0.01 \mathrm{M}_{\odot}$ Spitzer, 1978); and 2) more extreme star formation over a broader span of physical conditions such as metalicity, stellar density, galactic environment.

\section{Probing to the End of the IMF}

Advances in optical and infrared instrumentation on large groundbased telescope, as well as the Hubble Space Telescope have led to a number of IMF determinations that probe well into the brown dwarf regime. Several groups have derived sub-stellar IMF slopes in clusters that are flat, or falling, in log-mass units $d N / d \log m=m^{-\Gamma}$ with $\Gamma=0$ to 1 (e.g. Bouvier et al. 1998; Hillenbrand and Carpenter, 2000; Najita et al. 2000). While most regions appear to be consistent with each other, the Taurus dark cloud appears to have a statistically significant dirth of brown dwarfs compared to the Trapezium cluster (Briceno et al. 2002). Whether this is due to Taurus being deficient in brown dwarfs or the Trapezium being overabundant, is still not clear (Luhman, this volume).

A standard approach applied to the study of many young embedded clusters involves deep infrared imaging surveys along with follow-up infrared spectroscopy for as many cluster members as is practical (e.g. Greene and Meyer 1995; Luhman \& Rieke 1998). More recently, it has been possible to obtain IR spectra for low luminosity sources enabling astronomers to identify young brown dwarf candidates in large numbers (e.g. Luhman 1999; Wilking et al. 1999). One concern of these early studies was the use of photospheric absorption features to derive spectral types which might also be effected by surface gravity (lower in premain sequence "sub-giants" compared to high gravity dwarf standards). In a recent study of brown dwarf candidates in NGC 1333, Wilking et al. (2004) test whether their spectrophotometric reddening-independent $\mathrm{H}_{2} \mathrm{O} \mathrm{K}$-band index is effected by gravity. They find that any potential bias introduced by comparing PMS brown dwarf candidate spectra to dwarf star standards is of order the error inherent in the classification scheme. This is consistent with the work of Gorlova et al. (2003) who also find that $\mathrm{H}_{2} \mathrm{O}$ in the J-band depends weakly on surface gravity, making it a good temperature indicator for young late-type stars. How- 
ever, Gorlova et al. did find that the $1.25 \mu \mathrm{m}$ KI feature, along with the $2.2 \mu \mathrm{m} \mathrm{NaI}$ feature, is sensitive to surface gravity providing a possible tool to distinguish foreground and background stars from "sub-giant" PMS cluster members. We are currently following up this work with an approved Cycle \# 13 program on HST using NICMOS in slitless grism mode to obtain 1.1-1.9 $\mu \mathrm{m}$ spectra of brown dwarf candidates in NGC 1333. Our goal is to probe the IMF down below $10 \mathrm{M}_{J U P}\left(0.01 \mathrm{M}_{\odot}\right)$ in this intermediate density cluster (between Taurus and the Trapezium) to see if there is a correlation with the slope of the sub-stellar IMF.

Another curiousity observed in mass distributions that result from star formation processes, is the brown dwarf desert in the companion mass ratio distribution (CMRD). It is fairly well established from radial velocity surveys as well as seeing-limited common proper motion surveys (Udry et al. 2001; Hinz et al. 2001) that there is a dirth of brown dwarf companions compared to extrapolation of the CMRD of stellar or planetary companions. In fact, the CMRD from 0.1-1.0 $\mathrm{M}_{\odot}$ surrounding solar-type stars (Duquennoy \& Mayor, 1991; DM91) is consistent with having been drawn from a field star IMF, in stark contrast to the frequency of brown dwarf companions to sun-like stars compared to the sub-stellar IMF in the field (Ried et al. 1999). What about the companion mass ratio distribution surrounding young PMS stars? It has been known for some time that the binary frequency of $\mathrm{T}$ Tauri stars is high, particularly in low density star-forming regions such as Taurus-Auriga (Ghez et al. 1993; Leinert et al. 1993). However, it is only recently that researchers have begun to obtain multi-color infrared photometry and spatially-resolved IR spectra needed to derive mass estimates for faint companions to $\mathrm{T}$ Tauri stars. In fact, detailed comparisons of the CMRD between young clusters and the field are lacking.

In a NICMOS HST study of NGC 2024, Liu et al. (2003) describe how one must carefully consider completeness limits for faint companions as a function of angular separation and then compare the observations to results for field stars only over the appropriate mass ratios as a function of radius from the primary. They also consider what one might expect as a function of cluster age if soft-binaries are preferentially disrupted faster in higher density regions. Taking these effects into account, and combining their data with published results, they find a significant inverse correlation between binary frequency and stellar density. Fundamental survey work still needs to be done on the field star population as well as young star-forming regions, particular with the recent advances in adaptive optics on large telescopes. It will be important to know in this inherently three-dimensional observational problem whether: 1) the CMRD is a function of separation; and 2) whether the CMRD or 
the integrated period distribution is a function of primary mass. Although strong observational evidence to support the ejection hypothesis (e.g. Reipurth and Clarke, 2001) for the formation of free-floating brown dwarfs is lacking (e.g. Muench et al. 2002) it is curious to note that the one region deficient in brown dwarfs in the system IMF (Taurus) is also the lowest density star-forming region with the highest observed binary fraction.

What does the future hold in studying the sub-stellar IMF in starforming regions? Efforts continue to push down below the minimum mass for opacity-limited fragmentation in nearby young clusters. If there is a lack of objects below some cutoff mass, it will be hard to miss observationally, and particularly exciting if it depends on local conditions. Peale (1999) points out that if there are significant numbers of brown dwarfs below $0.01 \mathrm{M}_{\odot}$ in young clusters, it may suggest an IMF that varies over cosmic time as recent MACHO results rule out significant mass in the halo in objects $<0.01 \mathrm{M}_{\odot}$. One must be cautious in identifying extremely low mass objects in young clusters from photometry alone as there can be significant contamination in color-magnitude diagrams in deep surveys. We believe that spectroscopic follow-up is essential and spectroscopic diagnostics of surface gravity may be required in order to remove old cool field dwarfs from young cluster samples (Burgasser et al. 2004). We are working to identify a set of surface gravity diagnostics which could be used in filter-photometric imaging surveys to rapidly identify large samples of young late-type objects in star-forming regions. This effort complements recent work developing filter-photometric temperature diagnostics for very cool photospheres (e.g. Mainzer et al. 2003).

\section{Star Formation Extreme}

How does star formation in the solar neighborhood compare to global star formation in the Milky Way or even other galaxies? If one adds up the total number of high mass stars along the Gould's Belt within $1 \mathrm{kpc}$ of the Sun (de Zeeuw et al. 1999) and assumes a field star IMF,

then the integrated total mass of the system is about $3 \times 10^{5} \mathrm{M}_{\odot}$, comparable to a single "event" such as the R 136 cluster region in the LMC. Is the IMF in extreme star-forming regions such as R 136 (low metalicity, disturbed galactic morphology, high stellar density), or the galactic center (high metalicity, ambient pressure, and magnetic flux density) and comparable to that observed in the solar neighborhood? Can we probe further claims of unusual IMFs indirectly inferred from observations of starburst galaxies? While we may not be able to derive 
detailed distributions of the numbers of individual objects as a function of mass in these distant stellar systems, we can apply similar techniques to those described above to place limits on the ratio of high to low mass stars in these unusual regions that represent accessible extreme starforming environments.

\subsection{The Limits of Confusion}

In principle, one would like to conduct observational "experiments" where one could compare differentially results from observations of starforming regions varying one parameter at a time in a controlled way. However, such studies are thwarted by the limits one can achieve in sensitivity and spatial resolution for rare targets which tend to lie at the greatest distances. In particular, studies of the IMF in rich stellar fields are often confusion-limited rather than sensitivity-limited. For example, Andersen et al. (in preparation) find that NICMOS HST observations of the IMF toward R 136 in the LMC are confusion-limited at $>0.5 \mathrm{M}_{\odot}$. In this case, the spatial resolution afforded by large-ground based telescopes can help, but only if the point-spread function (PSF) is smooth and stable (Stolte et al. 2003).

In order to see how well one can do with currently operating adaptive optics (AO) systems on 6-10 meter telescopes, we performed a series of simulations using the observed PSF of the $6.5 \mathrm{~m}$ MMT with the new adaptive secondary mirror (Close et al. 2003). We considered the Trapezium cluster observations of Hillenbrand and Carpenter (2000) as input, and simulated what the Trapezium would look like at $5 \mathrm{kpc}$, $25 \mathrm{kpc}, 50 \mathrm{kpc}$, and $0.5 \mathrm{Mpc}$. As the cluster gets farther away, the residual uncorrected halo of the bright Trapezium stars dominates the background at progressively larger radii. At $5 \mathrm{kpc}$, one still reaches the sky background limit at 0.2 parsecs enabling one to easily detect objects at the sub-stellar boundary in reasonable integration times of a few hours. At $25 \mathrm{kpc}$ (Figure 2), one reaches the sky background limit $\times 5$ farther out still enabling one to measure a significant number of stars at $2.5 \mathrm{R}_{\text {core }}$ over the entire stellar mass range. At the core radius of the cluster inside of which most of the stellar population is located, one loses $\Delta H=6^{m}$ of sensitivity due to the background of uncorrected halo light from the bright stars. At $50 \mathrm{kpc}$, one does not hit the sky background limit until $4 \mathrm{R}_{\text {core }}$ and the sensitivity at the core radius is almost nine magnitudes worse than the natural background. At 0.5 $\mathrm{Mpc}$, the cluster is competely unresolved. We conclude that while it will be relatively straightforward to characterize the IMF down to the hydrogen burning limit for any Trapezium in the Milky Way galaxy with 
existing AO systems on 6-10 meter telescopes, achieving similar results in nearby galaxies will require higher spatial resolution afforded by the next generation of large telescopes (e.g. the Large Binocular Telescope).

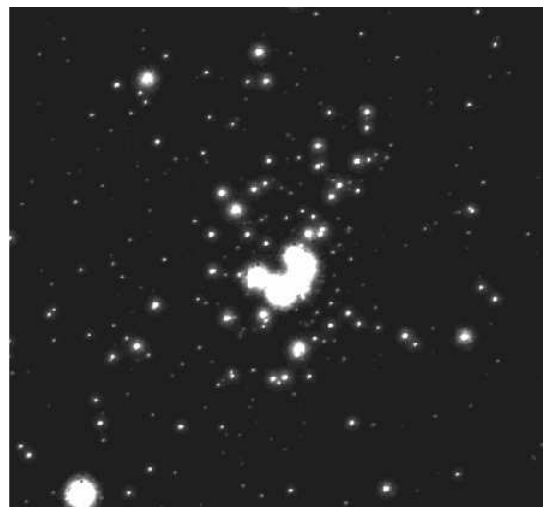

Figure 1. Simulations of the Trapezium cluster projected to a distance of $25 \mathrm{kpc}$ as it would appear observed with the MMT adaptive optics system. These observations would reach the natural background limit outside of 2.5 core radii enabling determination of the IMF over the full range of stellar masses.

Figer et al. (1999; this volume) have presented intriguing observations that suggest the IMF in the inner galaxy Arches cluster is flatter than the field star IMF over the mass range observed $\left(2-20 \mathrm{M}_{\odot}\right)$. In order to test whether or not the ratio of high to low mass stars in the Milky Way depends on metalicity, ambient ISM pressure, and/or magnetic field strength, we have begun a program using the ARIES camera in tandem with the MMT-AO system to image massive star forming clusters at high spatial resolution and sensitivity. Our first target is the distant luminous UCHII region W51 which contains over 130 stars $>10 \mathrm{M}_{\odot}$ (Okumura et al. 2000). Preliminary analysis of observations obtained in May, 2004 (Figure 2), indicate that we can reach the hydrogen burning limit in this cluster through $\mathrm{A}_{v}=30^{m}$ of extinction $\left(\mathrm{K}<21^{m}\right)$ in one hour of on-source integration time. We are currently exploring whether narrow-band IR imaging at $\mathrm{R}=100$ in $6-8$ filters can provide the necessary spectrophotometric information to estimate rough temperatures and surface gravities needed to distinguish foreground and background stars along this complex line of sight (Meyer et al. 1998).

\subsection{Unresolved Super-Star Clusters}

Some of the longest lasting claims of unusual IMFs come from observations of starburst galaxies with star-formation rates orders of magnitude higher than normal galaxies. Super-star clusters, recognized as globu- 


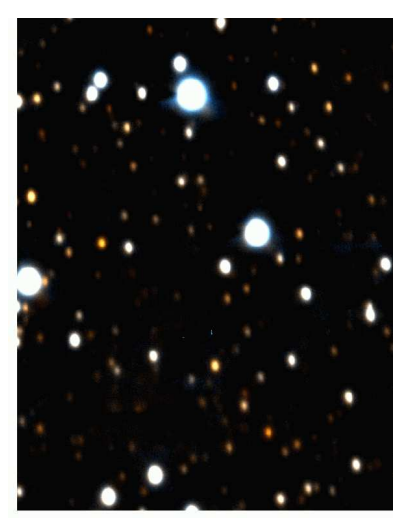

Figure 2. Observations of W51 in the H- and K-bands using the ARIES IR Camera and the MMT-AO system. Coadding 20 minutes of data resulted in images with 0.1 " FWHM in the $\mathrm{K}$-band. Preliminary results suggest that we will be able to reach the hydrogen-burning limit in the cluster in one hour of on-source integration time in the $\mathrm{K}$-band.

lar cluster analogues in the early stages of formation (O'Connell et al. 1994) have been found frequently within interacting starbursting galaxies. Mengel et al. (2002) used dynamical mass estimates from velocity dispersions to find significant variations in inferred mass-to-light ratios which they interpret as evidence for IMF variations from cluster to cluster in NGC 4038/39. Is there any way we could directly detect the low mass stars in these super-star clusters and constrain the ratio of high to low mass stars?

We have explored the contribution to the integrated $\mathrm{K}$-band light from very young low mass stars in super-star clusters $<10 \mathrm{Myr}$ old (Meyer and Greissl, 2004). While a main sequence population is dominated by the light from the highest mass stars because of the steepness of the main sequence mass-luminosity relation, in the PMS this M-L relation is much flatter perhaps rendering the low mass stars visible for a short time in a forming super-star cluster. Combining an assumed IMF (Salpeter, 1955 or Chabrier, 2003) with PMS evolutionary models (Siess et al. 2000) and predictions of Starburst99 for the main sequence, post-main sequence, and nebular contributions to the integrated light, we find that between $7-12 \%$ of the $\mathrm{K}$-band flux comes from young late-type stars depending on which IMF is assumed. Coupling these luminosity estimates with spectra appropriate for each object in the synthetic luminosity function, we have created model integrated spectra for the combined stellar population (Figure 3). Because these late-type stars are overluminous in the PMS, and because their spectra are dra- 
matically different, with strong broad absorption bands from $\mathrm{CO}$ and other molecules, we predict that in a super-star cluster $<10$ Myr (before the formation of the first red super-giants dominate the $\mathrm{K}$-band light of the cluster) one might detect directly the integrated light of the low mass stars as a 2-4\% absorption feature against the continuum. If one could use observations at centimeter and other wavelengths to models the contribution of nebular free-free emission to the $2 \mu \mathrm{m}$ continuum, the strength of the $\mathrm{CO}$ feature from late-type stars could provide a direct constraint on the ratio of high to low mass stars in these super-star clusters. The high signal to noise spectra $(>100)$ needed can be obtained at $\mathrm{R}=2000$ with NIRSpec on the Keck telescope in approximately five hours of integration time for the brightest candidate SSCs in NGC 4038/39 that are thought to be < 10 Myr (Frogel et al. 2003).

It must be cautioned that we have ignored some aspects of the problem in these simulations. Near-IR excess emission from disks surrounding the young low mass stars might dilute the expected absorption strength of the photospheric features in the IR (Meyer et al. 1997). However, because PMS stars have sub-giant surface gravities, we are also underestimating the strength of $\mathrm{CO}$ feature which is surface gravity sensitive (Kleinmann and Hall, 1986). We do not argue that these simulations are a perfect representation of what one should expect from the integrated light of super-star clusters. Nevertheless, we draw inspiration from Salpeter (2002), who, while musing about the longevity of his 1955 paper, suggested that it is not important for all the assumptions in a calculation be correct, but that the largest uncertainties should tend to cancel!

\section{Conclusions}

Our conclusions can be briefly summarized at follows: 1) the stellar IMF observed in nearby star-forming regions is broadly consistent with the field star IMF and does not vary strongly with local conditions (Meyer et al. 2000); 2) sub-stellar objects contribute less than $10 \%$ of the dynamical mass of young stellar populations (Wilking et al. 2004) and this fraction might vary from region to region (Briceno et al. 2002); 3) there may be a connection between the binary fraction in a young cluster, its stellar density, and the observed system IMF (Liu et al. 2003); 4) spectroscopic estimates of temperature and surface gravity are needed in order to survey down below the expected minimum mass for fragmentation in nearby molecular clouds (Gorlova et al. 2004); 5) while existing AO systems on 6-10m telescopes should enable us to probe down to the hydrogen burning limit throughout the Milky 


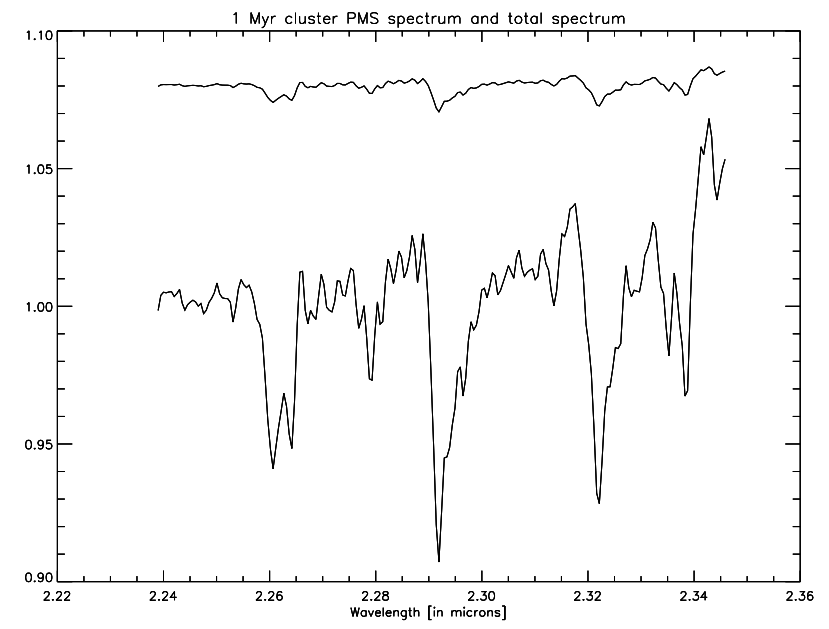

Figure 3. The total integrated K-band spectrum of a 1 Myr old $10^{6} \mathrm{M}_{\odot}$ unresolved super-star cluster (top) and the associated spectrum of the PMS stars only (bottom). In a few hours of integration time on a 6-10 meter telescope, we should be able to detect the signature of the late-type low mass stars (2-4\% absorption features against the continuum) in candidate super-star clusters in NGC 4038/39.

Way galaxy (e.g. W51), it will be extremely difficult to reach this limit even in the local group without higher spatial resolution afforded by the next generation of very large telescopes such as the LBT; and 6) it may be possible to detect directly the low mass stellar component in the high SNR spectrum of an integrated super-cluster in nearby interacting galaxies placing constraints on the ratio of high to low mass stars (Meyer and Greissl, 2004).

\section{References}

Bouvier, J., Stauffer, J.R., Martin, E.L., Barrado y Navascues, D., Wallace, B., Bejar, V.J.S. 1998, A\&A, 336, 490

Briceno, C. Luhman, K.L., Hartmann, L., Stauffer, J.R., Kirkpatrick, J.D. 2002, ApJ, 580,317

Burgasser, A.J, Kirkpatrick, J.D, McGovern, M.R., McLean, I.S., Prato, L., Reid, I.N. 2004, ApJ, 604, 827

Chabrier, G. 2003, ApJL, 585, 133

Close, L.M. et. al. 2003, ApJ, 599, 537

Duquennoy, A., Mayor, M. 1991, A\&A, 248, 485

Figer, D., Kim, S., Morris, M., Serabyn, E., Rich, R., McLean, I. 1999, ApJ, 118, 2327

Ghez, A.M., Neugebauer, G., Matthews, K. 1993, AJ, 106, 2005

Gorlova, N.I., Meyer, M.R., Rieke, G.H., Liebert, J. 2003, 593, 1074

Greene, T.P., Meyer, M.R. 1995, ApJ, 450, 233 
Hillenbrand, L.A., Carpenter, J.M. 2000, ApJ, 540, 236

Hinz, P.M., Hoffmann, W.F., Hora, J.L. 2001, ApJL, 561, 131

Kassin, S.A., Frogel, J.A., Pogge, R.W., Tiede, G.P., Sellgren, K. 2003, AJ, 126, 1276

Kleinmann, S.G., Hall, D.N.B. 1986, ApJS, 62, 501

Leinert, Ch., Zinnecker, H., Weitzel, N., Christou, J., Ridgway, S.T., Jameson, R., Haas, M., Lenzen, R. 1993 A\&A, 278, 129

Liu, W.M., Meyer, M.R, Cotera, A.S., Young, E.T. 2003, AJ, 126, 1665

Luhman, K.L. 1999, ApJ, 525, 466

Luhman, K.L., Rieke, G.H. 1998, ApJ, 497, 354

Mainzer, A.K., McLean, I.S. 2003, ApJ, 597, 555

Mengel, S., Lehnert, M.D., Drob, D.P., Porter, H.S. 2002, A\&A, 383, 137

Meyer, M.R., Calvet, N., and Hillenbrand, L. 1997, AJ, 114, 198

Meyer, M.R., Edwards, S., Hinkle, K.H., Strom, S.E. 1998, ApJ, 508, 397

Meyer, M.R. et. al. 2000, in Protostars and Planets IV, ed. Mannings, V., Boss, A.P., Russell, S.S. (Tucson: University of Arizona Press), p. 121

Meyer, M.R., and Greissl, J. 2004, ApJ, submitted.

Muench, A.A., Lada, E.A., Lada, C.J., Alves, J. 2002, ApJ, 573, 366

Najita, J.R., Tiede, G.P., Carr, J.S. 2000, ApJ, 541, 977

O'Connell, R.W, Gallagher, J.S., Hunter, D.A. 1994, AJ, 108, 1350

Okumura et. al. 2000, ApJ, 543, 799

Peale, S.J. 1999, ApJL, 524, 67

Reid, I.N., et al. 1999, ApJ, 521, 613

Reipurth, B., Clarke, C. 2001, AJ, 122, 1508

Salpeter, E.E. 2002, ARA\&A, 40, 1

Salpeter, E.E. 1955, ApJ, 121, 161

Siess, L., Dufour, E., Forestinin, M. 2000, A\&A, 358, 593

Spitzer, L. 1978, Physical Processes in the Interstellar Medium, (Wiley: New York).

Stolte, A., Brandner, W., Grebel, E.K., Figer, D.F., Eisenhauer, F., Lenzen, R., Harayama, Y. 2003, Msngr, 111, 9

Udry et. al. 2001. A\&A.

Wilking, B.A., Greene, T.P., Meyer, M.R. 1999, AJ, 117, 469

Wilking, B., Meyer, M., Greene, T., Mikhail, A., Carlson, G. 2004, AJ, 127, 1131

de Zeeuw, P.T., Hoogerwerf, R., de Bruijne, J.H.J., Brown, A.G.A., Blaauw, A. 1999, AJ, 117,354

\section{Acknowledgments}

We would like to thank the meeting organizers for providing such a stimulating get-together in such a lovely place where we could share ideas on this work with such generous and talented colleagues. We congratulate Prof. Salpeter on his lifetime of important achievements in astrophysics and wish him many more years of good health and happiness. This work was generously supported by a Cottrell Scholar's Award to MRM from the Research Corporation and NASA grant HST13-9846. 